\title{
El uso de dispositivos móviles como estrategia educativa en la universidad
}

\section{(The use of mobile devices as an educational strategy at university)}

Gustavo Carlos Mangisch Moyano

María del Rosario Mangisch Spinelli

Universidad Católica de Cuyo, UCCUYO (Argentina)

DOI: http://dx.doi.org/10.5944/ried.23.1.25065

\section{Cómo referenciar este artículo:}

Mangisch Moyano, G. C., y Mangisch Spinelli, M. R. (2020). El uso de dispositivos móviles como estrategia educativa en la universidad. RIED. Revista Iberoamericana de Educación a Distancia, 23(1), pp. 201-222. doi: http://dx.doi. org/10.5944/ried.23.1.25065

\section{Resumen}

Las Tecnologías de la Información y la Comunicación han causado un gran impacto en todas las actividades humanas. Con la irrupción de los teléfonos inteligentes se ha acelerado un proceso profundo de transformación en la disponibilidad y el acceso al conocimiento. La educación, y particularmente la universidad, están interpeladas por estas nuevas tecnologías que utilizan frecuentemente tanto los alumnos como los docentes para el funcionamiento de muchas actividades de su vida personal, pero: ¿logran incorporase adecuadamente a la práctica educativa? La investigación se realizó a través de una metodología cualitativa de carácter descriptivo, que recoge el testimonio del uso que se hace de los dispositivos móviles en la universidad y los contextos que condicionan estos comportamientos. El estudio aporta información relevante sobre las aplicaciones que le dan los profesores y alumnos de la universidad a sus dispositivos móviles, vinculadas con los procesos de enseñanza y aprendizaje, y aporta información significativa sobre el impacto y el potencial uso de las TIC en la educación universitaria, para favorecer la política en materia de desarrollo tecnológico y digital en la universidad y contribuir a las decisiones en materia de desarrollo e inversión del área tecnológica. En la perspectiva del debate y luego de un análisis interpretativo-crítico de la información obtenida en el trabajo, se sugieren algunos hallazgos identificados en el estudio, propuestas de mejora que permitan optimizar los planes de virtualización y el uso de dispositivos móviles y posibles nuevos interrogantes que se abren a partir de la investigación.

Palabras clave: enseñanza superior; tecnología de la información; sistemas multimedia. 


\begin{abstract}
Information and Communication Technologies have made a great impact in all human activities. The arrival of smartphones has sped up the process of profound transformation in knowledge availability and accessibility. Education, higher education in particular, is highlighting the issue of new technologies which both students and teachers frequently use in their personal lives. However, are they successfully incorporated in educational practice? The current research has been conducted using a qualitative methodology of a descriptive character, which gathers the testimony of the use of mobile devices in the university and the contexts that determine this behavior. The study supplies relevant information about the use that teachers and students make of their mobile devices related to the teaching and learning processes, and supplies significant information about the impact and potential use of ICT in higher education in order to pursue policies related to technological and digital development in universities as well as contribute to the decisions made in technological development and investment. From the perspective of the debate and a further interpretative and critical analysis of the data gathered in the study, some of the identified findings are provided. These are proposed improvements that allow for the optimization of virtualization plans and the use of mobile devices, as well as new questions which arise from the investigation.
\end{abstract}

Keywords: higher education; information technology; multimedia systems.

En el mundo están sucediendo cosas increíbles que producen avances científicos y tecnológicos impensables desde hace algunas décadas atrás (Solano Barcenas, 2015). En esta "nueva era" de cambios profundos y vertiginosos, el rasgo distintivo tiene que ver con el conocimiento abundante y accesible y, literalmente, al "alcance de la mano" (o de los dedos) de cualquier persona conectada a Internet, con algún dispositivo de procesamiento digital, fijo o móvil. Se denomina a esta sociedad como "sociedad del conocimiento" (Cassany, 2013). Se pone el acento no tanto en la retención del conocimiento, sino en la competencia para interactuar con él en la creación de nuevos saberes y habilidades. En esta perspectiva, la educación está llamada a desarrollar competencias que permitan la gestión del conocimiento.

Algunos autores afirman que la educación en todos sus niveles ha venido sobreviviendo a los cambios que se operaron en otros sectores de la sociedad y que, en lo fundamental, hay pocas diferencias entre lo que enseñamos hoy y cómo lo enseñamos, y lo que se enseñaba hace ciento cincuenta años. Indican además que el problema central de la educación no es que ya no sea como antes; el problema es que en muchos casos sigue siendo igual que antes (Adell Segura y Castañeda Quintero, 2010). Investigaciones recientes sostienen que las TIC van a generar un cambio de paradigma (Aguiar, Velázquez y Aguiar, 2019) en donde la organización del conocimiento ya no será por disciplinas sino por problemas, temáticas o focos. También va a cambiar la evaluación, que ya no será a través de exámenes sino por “desempeños”. Los libros serán sólo alternativos; se utilizarán páginas web y redes 
sociales en las que se proponen proyectos creados por los profesores y por los alumnos. Se priorizará la autoevaluación, la evaluación entre pares y la evaluación externa. Se conformarán grupos de profesores que enseñan en una estrategia común porque se trabajarán las dimensiones no cognitivas, la responsabilidad y la autodirección.

Asistimos a una transformación en los modos en que se produce y circula el conocimiento, acceso a investigaciones de diferentes países y universidades, modificaciones en los vínculos entre expertos y novatos, y en los formatos de la innovación y cambios en la toma de decisiones. Por otro lado, las nuevas generaciones que se forman en la universidad aportan al escenario educativo un conjunto de prácticas y saberes ligados con un nuevo ecosistema comunicativo caracterizado por la multiplicación y concentración de las tecnologías comunicativas e informacionales.

En el año 2007, Steve Jobs realizó la presentación del primer teléfono inteligente que cambiaría la historia de la comunicación en todo el planeta, acelerando un proceso de transformaciones culturales que ya había anticipado 10 años antes el acceso a Internet a través de la World Wide Web (WWW). Por lo novedoso del tema, no es posible identificar en el estado del arte, experiencias e información sobre esta investigación que sean anteriores a los últimos 10 años. Pero a la vez, en este corto tiempo existen numerosos estudios académicos y científicos sobre la temática (Mao, Ifenthaler, Fujimoto, Garavaglia y Rossi, (2019), especialmente para el uso de estas tecnologías en la educación Primaria y Secundaria. El profesor Nicholas Negroponte desde el Massachusetts Institute of Technology (MIT), promovió a nivel mundial lo que se denominó el Proyecto OLPC (del inglés "One Laptop per child" - Una computadora por niño). Su objetivo era fabricar computadoras portátiles de bajo costo que se ofrecerían a países en vías de desarrollo para entregar a todos los niños y jóvenes del sistema educativo (Bender, Kaner, Cornish y Donahue, 2012). Este proyecto estimuló a muchas empresas privadas a producir también laptops y tablets a bajo costo y varios países estuvieron entregando una computadora por cada alumno de todas sus escuelas. El proyecto más difundido lo ha desarrollado la República Oriental del Uruguay, comenzando con todos los alumnos de las escuelas primarias de ese país, que denomina "Plan Ceibal" (Rivoir, 2013). En la Argentina se llevó adelante desde el Gobierno Nacional un proyecto similar al de Uruguay bajo la denominación de Conectar-Igualdad (Fontdevila, 2011), que llegó a distribuir más de cinco millones de equipos, pero en todas las escuelas del Nivel Medio de gestión estatal. Se destacan también la Ciudad Autónoma de Buenos Aires con el "Plan S@rmiento", la provincia de San Luis con el "Plan Todos los Chicos en Red" y el "Programa Joaquín V. González" de La Rioja. Estos desarrollos han generado una importante producción de bibliografía y de investigaciones sobre el tema. Sin embargo, es sensiblemente menor tanto la implementación de dispositivos móviles para el estudio en las universidades y la edición de bibliografía, como la producción de estudios académicos sobre su impacto.

Sabemos bastante para qué sirven los teléfonos inteligentes (Deloitte, 2017) y en particular, el uso que le dan los jóvenes a sus celulares (Lozano Ramírez, 2019). 
Son muchas las instituciones que están invirtiendo en tecnología y desarrollando contextos para su utilización. También existen nuevos estudios sobre la utilización de dispositivos móviles en las universidades, alguno de los cuales sirvieron de inspiración para el presente trabajo. (Fernández Muñoz y García García, 2016; Henríquez Ritchie, Organista Sandoval y Lavigne, 2013; Hernández Ramos y Martínez Abad, 2017; Ma'azer Al Fawareh y Jusoh, 2017).

Varios especialistas en educación sostienen que existe una velocidad diferente entre la apropiación de las tecnologías de la información y la comunicación (TIC) en las universidades y su utilización por parte de los alumnos (Lagunes Domínguez, 2019). Este fenómeno producido por los vertiginosos cambios operados en los últimos años, hacen necesario explorar, desde una perspectiva investigativa, cómo ellos están afectando a los actores del proceso de enseñanza y aprendizaje (Almenara y Osuna, 2015).

Frente al impacto de las TIC en todas las áreas del quehacer humano, en el caso de la educación del nivel superior, es importante investigar y aportar información significativa sobre el uso que le dan los profesores y alumnos universitarios, para favorecer y focalizar la política en materia de desarrollo tecnológico y digital de la universidad.

\section{METODOLOGíA}

A pesar de que ha pasado poco más de una década desde que se presentó el primer teléfono inteligente (Schlender y Tetzeli, 2016), su utilización ha transformado muchas prácticas que venían acompañando a la humanidad durante siglos, tanto en el área de las comunicaciones entre las personas como en la obtención de información, la lectura, el ocio, la movilidad, y muchas otras actividades más. La hiperconectividad (Marquez, 2019) que posibilitan los dispositivos móviles y su portabilidad permiten acceder a grandes volúmenes de información, convirtiéndose en un recurso muy necesario para la vida cotidiana, que se proyecta también como de alto interés para la educación. Si bien algunos describen críticas al impacto que genera el uso de teléfonos inteligentes en las aulas (Pinos Paredes, 2018), interesa analizar en qué medida y con qué aplicaciones, tanto docentes como alumnos, están utilizando estos dispositivos en los procesos de enseñanza y aprendizaje en la universidad (Lagunes Domínguez, 2019).

Algunas preguntas que surgen del planteo del problema son: ¿Está instalada la preocupación por el uso de dispositivos móviles en las clases de la universidad? ¿Qué cantidad de alumnos y docentes utilizan dispositivos móviles en el proceso de enseñanza y aprendizaje dentro de la universidad? ¿Qué tipo de aplicaciones y herramientas se utilizan? ¿Qué tipo de estrategias tiene la universidad para promover el uso de las TIC en el aula? ¿Qué se podría hacer además de lo que se está haciendo? 


\section{Objetivo del trabajo}

El presente trabajo busca indagar sobre el uso que les dan a los dispositivos móviles los alumnos y profesores de la universidad, el contexto en que los enmarcan y las posibles acciones para un mayor aprovechamiento de los mismos (Mangisch, 2018).

\section{Objetivos específicos}

1. Observar cuál es el uso, la frecuencia con que se utilizan, y el volumen de alumnos y docentes de la universidad que utilizan dispositivos móviles para fines educativos.

2. Identificar las principales aplicaciones que la población estudiada utiliza en sus dispositivos móviles.

3. Indagar sobre las políticas y la infraestructura para el desarrollo de la plataforma virtual y tecnológica-comunicacional de la universidad.

4. Proponer estrategias para la promoción de un mayor uso de tecnologías en las clases, particularmente de los dispositivos móviles.

\section{Tipo de Investigación}

Cualitativa y cuantitativa de carácter descriptiva.

\section{Características de la muestra}

La población está conformada por directivos dela universidad, docentes y alumnos de las carreras de Derecho y Ciencias Sociales, Filosofía y Humanidades, Ciencias Económicas, Ciencias Químicas y Tecnológicas, Ciencias Médicas y Educación, en los niveles de pregrado, grado y posgrado, pertenecientes a la Universidad Católica de Cuyo, en Argentina.

\section{Selección de los informantes}

Se determinó al grupo de alumnos y docentes para la encuesta basándose en un número que estima un margen de error en torno al $5 \%$ con niveles de confianza entre el 90 y $95 \%$.

Se realizaron dos focus group con docentes y dos con alumnos, a los cuales se convocó en forma aleatoria.

Las entrevistas en profundidad se realizaron con el Rector, el responsable del área de Educación Virtual y la Secretaria de Extensión. 


\section{Técnicas para la recolección de datos}

\section{Encuestas}

La información que se recogió a través de encuestas se vinculaba a tres aspectos particulares para este estudio:

1. Conocer la opinión de los docentes de la universidad referida al uso de las TIC y, más específicamente, al uso de dispositivos móviles (tabletas o teléfonos inteligentes).

2. Conocer la opinión de los alumnos de la universidad referida al uso de las TIC y, más específicamente, al uso de dispositivos móviles (tabletas o teléfonos inteligentes).

3. Determinar los aciertos y las dificultades que enfrentan en la utilización de estos dispositivos móviles.

\section{Entrevistas en profundidad}

A partir de la tabulación de las encuestas y posterior análisis de los datos recopilados, se planificaron algunas entrevistas en profundidad con el objetivo de ahondar en la información obtenida y recoger algunas opiniones adicionales que nos permitieron un análisis más profundo sobre cómo impactó el liderazgo en el proceso de mejora.

\section{Focus Group (FG)}

Como corolario en la búsqueda de datos relevantes a partir del testimonio y opinión de las personas, se realizaron reuniones para profundizar la información obtenida hasta ese momento.

\section{Observación directa}

Se realizó una actividad de observación de algunas clases.

\section{RESULTADOS}

El cuestionario de la encuesta fue enviado al alumnado y al profesorado a través de una aplicación de Internet con la intención de que el número de respuestas que se obtendrían serían en sí mismas un indicador de interés para la investigación. De los aproximadamente 8400 alumnos que asisten a la universidad, respondieron a la encuesta 358 (4,3\%) con la siguiente distribución: 
Figura 1. Alumnos por tipos de carrera

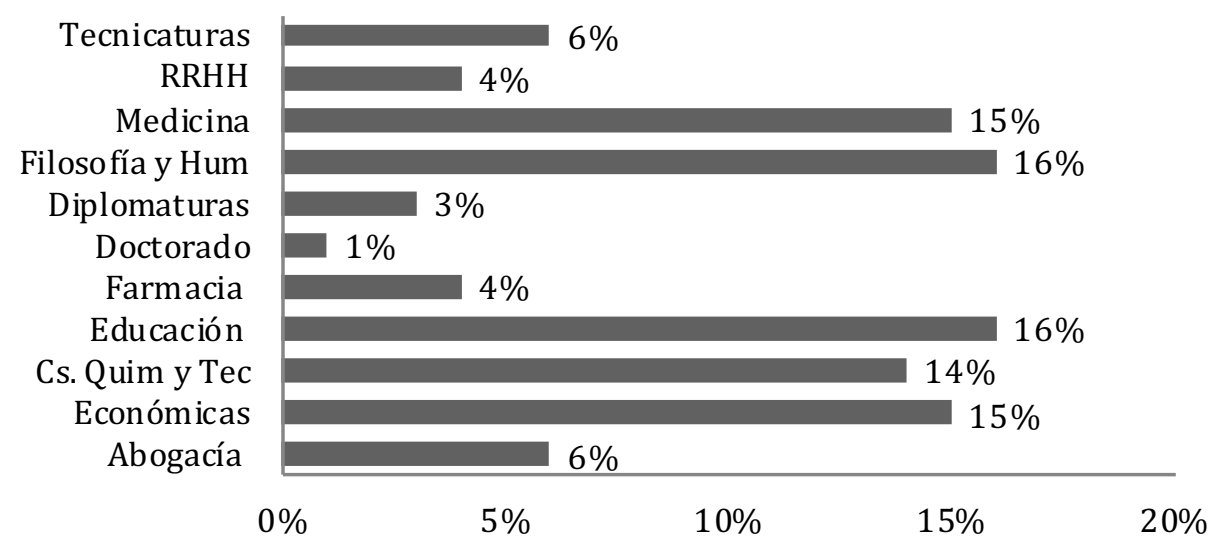

De los 1078 docentes, lo hicieron 104 (9,6 \%) con la siguiente distribución:

Figura 2. Docentes por tipos de carrera

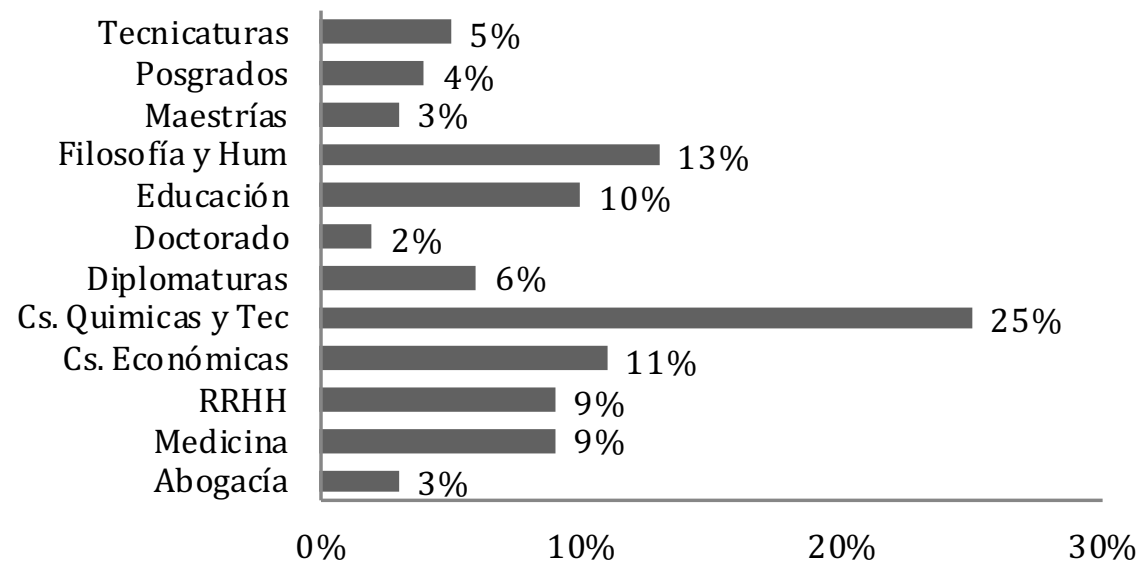

Para cualquier proyecto que intente desarrollar estrategias con la incorporación de tecnologías a la actividad educativa, la disponibilidad de equipamiento es un prerrequisito fundamental. Tanto en los FG como en las entrevistas se pudo verificar por la opinión de los participantes que un alto porcentaje de los encuestados declaran tener dispositivos móviles con acceso a Internet (Anderson y Jiang, 2018). 
En las encuestas, el 96,9\% de los alumnos y el 97,2 \% de los profesores declaran tener teléfonos inteligentes. En las entrevistas y FG el 100 \% de los participantes expresan que poseen teléfonos inteligentes.

Figura 3. Tenencia de teléfonos inteligentes

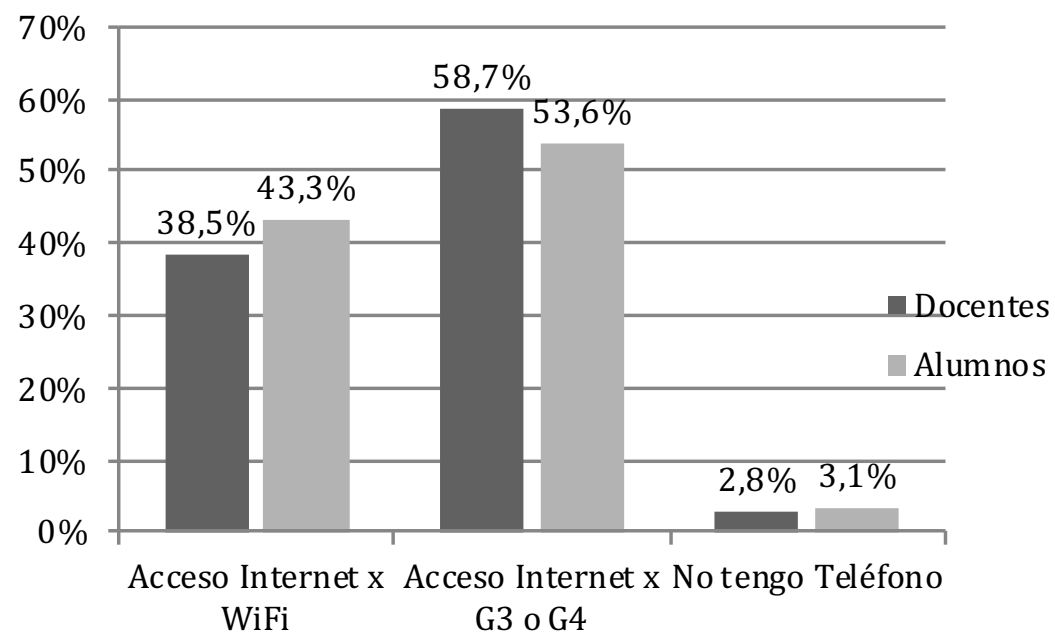

Si bien es conocida la masificación en el uso de dispositivos móviles en todo el mundo, la investigación corrobora la disponibilidad de equipos en prácticamente la totalidad del profesorado y de sus alumnos.

Otra información que surgió de la encuesta a los docentes es que el $43 \%$ de los que respondieron indicaron que enseñan en la universidad hace menos de cinco años. El $90 \%(71 \%+19 \%)$ de los profesores declaran haber recibido algún tipo de capacitación durante los dos últimos años.

Figura 4. Antigüedad docente

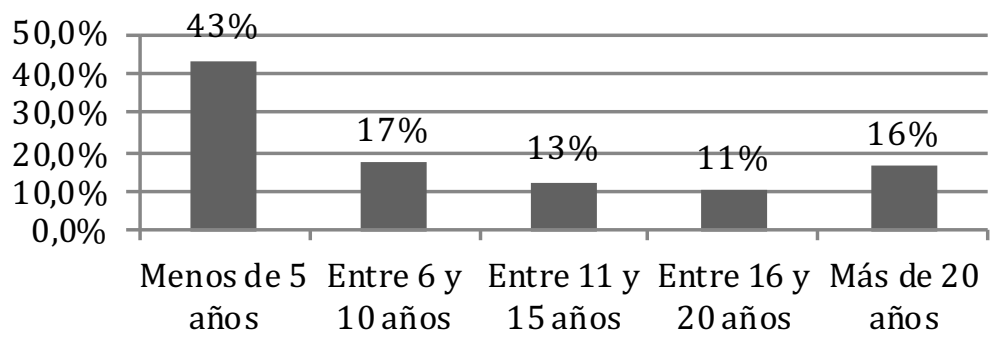


Figura 5. Capacitación

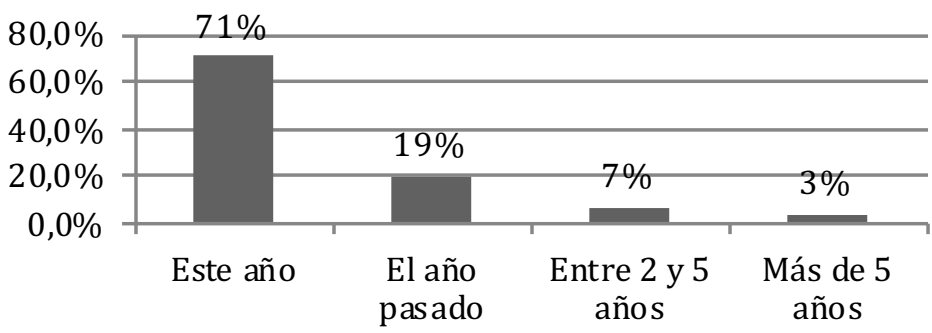

Del $68 \%$ que dice haber tomado cursos a distancia, solamente el $12 \%$ ha sido tutor o profesor de propuestas con esta modalidad. Si este dato se vincula con la cantidad de docentes que manifestaron en la investigación la necesidad de recibir capacitación en el uso de tecnologías, parece evidente la posibilidad de que la universidad pueda proponer con éxito itinerarios formativos en temas vinculados con el uso de TIC en educación.

En el caso de los alumnos, el 75 \% no ha tenido experiencia ni ha tomado cursos en la modalidad a distancia. Cuando se les pregunta a los docentes sobre su percepción acerca de sus conocimientos teóricos sobre el impacto tecnológico en la cultura actual, el $86 \%$ de ellos creen que son buenos, muy buenos o excelentes (46 \%, $29 \%$ y $11 \%)$. Cuando se refieren específicamente a la aplicación de las TIC en la universidad, desciende algunos puntos (82 \%).

En la comparación entre profesores y alumnos sobre la pregunta referida a la experiencia en la aplicación de las TIC a la educación, el 78 \% de los primeros indican tener experiencia entre buena a excelente, mientras que para los segundos es el $67 \%$.

Si cruzamos esta información con datos de las entrevistas y las observaciones y los referidos en la encuesta al uso y frecuencia de aplicaciones por parte de los usuarios, parecería haber una sobreestimación o, al menos, una distancia entre lo que se cree y lo que se hace. Convendría hacer un estudio con parámetros más específicos y con indicadores y marcas para identificar el verdadero background teórico como punto de partida para la definición de una estrategia de capacitación.

La evaluación sobre la infraestructura tecnológica que ofrece la universidad indica que para el $59 \%$ de los alumnos es entre buena a excelente (31\%, 22 \% y $6 \%$ ), y que para el $73 \%$ de los profesores es entre buena a excelente (36\%, $28 \%$ y $9 \%$ ). 
Figura 6. Calificación infraestructura

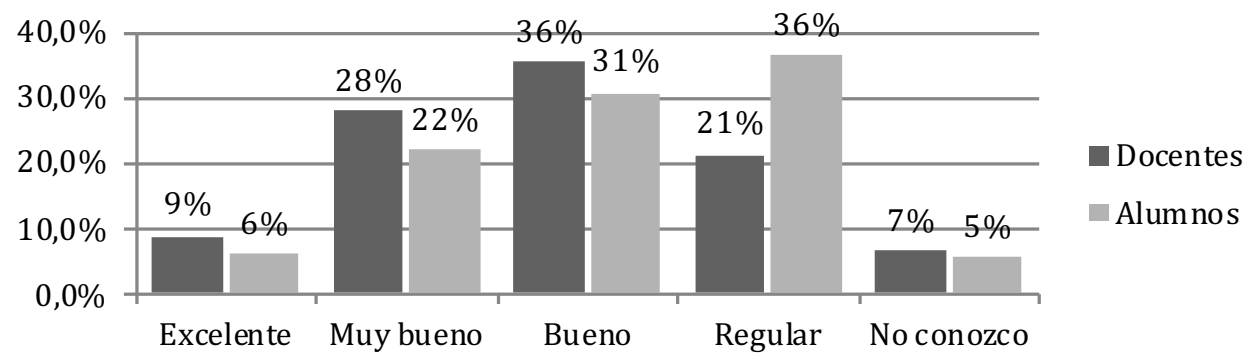

Si bien la opinión del $73 \%$ de los docentes y el $59 \%$ de los alumnos pareciera aceptar como suficiente la disponibilidad de recursos que ofrece la universidad, esta aseveración contrasta con los testimonios recogidos en los FG y en las entrevistas, que evaluaron negativamente la disponibilidad de recursos dentro del campus.

La principal demanda, tanto de docentes como alumnos, está planteada en una mayor disponibilidad de conectividad y acceso a la red en las instalaciones de la universidad.

El $75 \%$ de los profesores y el $81 \%$ de los alumnos dicen utilizar el celular para navegar por Internet o gestionar redes sociales. El 36\% lee libros y el $22 \%$ toma notas. También en este punto es notable el alto uso que hacen profesores y alumnos fuera de la actividad educativa.

Se agrega a la disponibilidad, la familiaridad en el uso de aplicaciones de celulares para varias cuestiones de la vida privada.

El reclamo de mayor desarrollo de aplicaciones en el ámbito de estudios indicaría que una propuesta para incrementar el uso de celulares en las aulas será bienvenida.

El $57 \%$ de los profesores cree que la tecnología transformará la educación, mientras que sólo el $38 \%$ de los alumnos lo cree. Es importante el dato del uso que le dan los estudiantes al celular en su vida cotidiana, ya que la gestión y participación de redes sociales con el $80 \%$ de las respuestas es el principal uso, seguido por la navegación en Internet con el 75 \%. Recién en el tercer lugar con el 45 \% de respuestas aparece el uso del celular para hacer y recibir llamadas.

En cuanto al uso de los dispositivos móviles en la universidad, el $50 \%$ de los docentes y el $49 \%$ de los alumnos dicen utilizarlos para hablar y mandar mensajes por WhatsApp. El 16\% de los primeros dice usarlo para las clases y el $32 \%$ de los alumnos declaran usarlos para estudiar.

Cuando se les pregunta por la frecuencia de uso, la aplicación más utilizada diariamente es el WhatsApp con el 99\%, seguida por la navegación en Internet con $81 \%$ y el Email con el $55 \%$. 
Figura 7. Frecuencia de uso

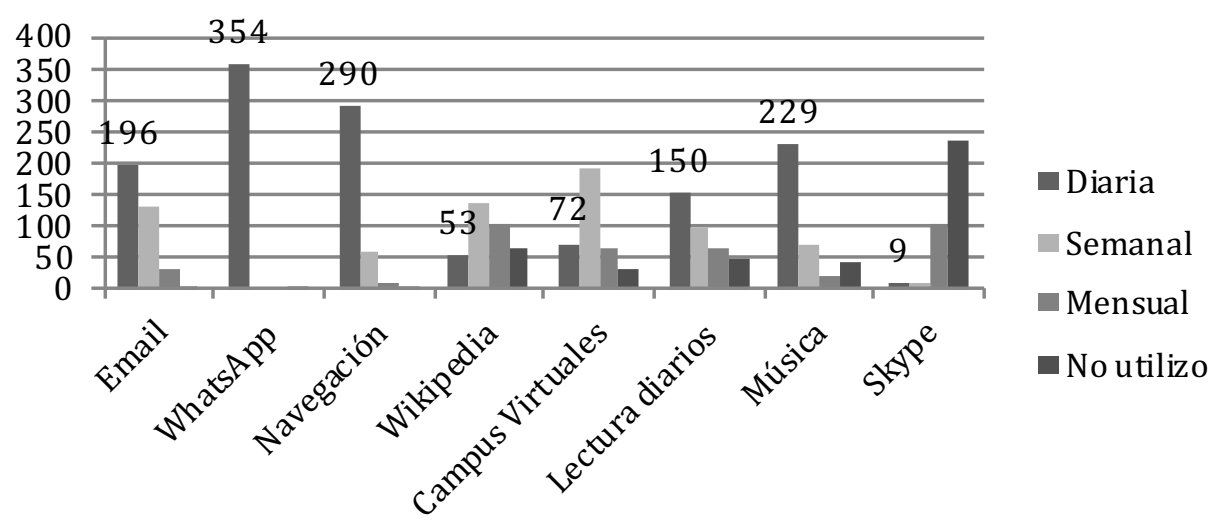

De las entrevistas y los grupos se percibe que es bajo el porcentaje de los docentes que usan aplicaciones y herramientas que podrían contribuir a la utilización de dispositivos dentro de la universidad, inclusive aquellas especialmente diseñadas para el uso en la universidad como es el caso del Moodle que llega al $31 \%$ de utilización en docentes y baja al $20 \%$ en el caso de los alumnos.

A continuación, se detallan los principales aspectos y características en cuanto al desarrollo del contexto digital en la universidad que surgieron de los participantes en los FG y/o de las entrevistas en profundidad.

\section{Incorporación de la educación virtual en la universidad}

La educación virtual en los últimos años en la universidad se ha ido consolidando muy poco y lentamente (Arkorful y Abaidoo. 2014). Sólo se lleva adelante por algunos docentes que utilizan la plataforma Moodle y por cursos de parte de algunas unidades académicas con fines muy específicos, generalmente como oferta de formación de postgrado. La mayoría de las propuestas desarrolladas en la plataforma de la universidad se refieren al desarrollo de actividades virtuales de acompañamiento y sostén a lo que son las tareas presenciales, sobre todas las cátedras de grado.

\section{Receptividad de las propuestas de Educación virtual}

Por lo general los docentes han recibido estas propuestas de educación virtual con adhesión relativamente baja. Los docentes que se acercan voluntariamente o por curiosidad, trabajan y van creativamente incorporando instrumentos y recursos a la plataforma; y también es ese espíritu que le transmiten a los alumnos y el 
entusiasmo y aprovechamiento de la herramienta. Hay docentes que utilizan Moodle como repositorio de información y no se interesan en motivar a los estudiantes. El mismo docente genera el espacio virtual pero no lo usa como medio de comunicación con sus estudiantes.

\section{Principales desafíos que enfrenta la universidad frente a las TIC}

Una de las consecuencias positivas de desarrollar educación virtual como modalidad de enseñanza de algunos profesores es que se le da entidad y visibilidad a la digitalización aplicada a la educación superior como modalidad de trabajo. Permite reconocerla como posibilidad real entre las estrategias de enseñanza y aprendizaje. Contribuye al planteo de consignas de trabajo, de evaluación o de seguimiento, se incorpora a la práctica cotidiana, tiene en cuenta las devoluciones de lo que se ha trabajado en seguimiento particular del desarrollo de cada uno de los estudiantes. El docente que efectivamente está usando la educación virtual es aquel que le da cabida y le da cierta consistencia a su trabajo; de otro modo no deja de ser un recurso más y totalmente descartable. En la referencia a los desafíos más importante que enfrenta la universidad frente a las TIC se destacó: convencer a autoridades, directivos, docentes de la necesidad de la educación a distancia; toma de decisión y acompañamiento de los respectivos equipos de gestión; necesidad de formación y desarrollo de competencias a tal fin; capacitación en masa de docentes en esta modalidad; construcción de un centro de educación a distancia con áreas específicas; equipos técnicos que lideren la capacitación y faciliten la puesta en marcha ayudando a los docentes a resolver problemas técnicos y de conectividad (Kostakos, 2018).

\section{Importancia de la infraestructura tecnológica para la educación virtual}

Es clave y de fundamental importancia tanto la infraestructura como el equipo técnico, tanto la capacitación como el insumo fundamental del proyecto. Sin esto no sería posible impartir educación a distancia. Otro aspecto importante común a todas las instituciones es garantizar la calidad y la regularidad del encuadre tecnológico dentro del campus, que haya buena señal de wifi y que haya recursos de accesibilidad para profesores y estudiantes. Esta mejora de la conectividad se visualiza como uno de los prerrequisitos que es constante y que va más allá de cuáles sean las intenciones de la universidad con relación a ser o no una universidad que tenga oferta académica a distancia. 


\section{Estrategias de mejora de la plataforma de conectividad}

Es clara la convicción de que la infraestructura tecnológica es importantísima, sobre todo cuando se quieren desarrollar dentro de las clases presenciales actividades que tengan que ver con la búsqueda de información en Internet o sostener dentro de lo que es cualquier propuesta virtual instancias sincrónicas de comunicación, como pueden ser las videoconferencias o chats. Del estudio se deduce que la infraestructura tecnológica es vital y siempre hay que estar al tanto e inclusive, si es posible, un paso adelante. Es importante cubrir todas las posibilidades de las demandas que puedan surgir sobre educación virtual.

\section{Uso de los dispositivos móviles}

Unánimemente se sostiene que el uso de los dispositivos móviles es una herramienta vital fuera del aula y muy útil para todas las actividades, ya que tanto docentes como alumnos se encuentran durante las jornadas laborales y en su vida personal utilizándolos frecuentemente. El uso de las TIC, los dispositivos móviles, las redes sociales y los buscadores en los próximos años marcará el sentido de la comunicación y seguramente su utilización deberá ser motivada y promovida por los propios docentes e investigadores, ya que constituirán los principales canales de acceso a la información y a la innovación (López Hernández y Silva Pérez, 2016). No obstante, debemos ser conscientes de que demandan una nueva pedagogía y un clima de auténtica horizontalidad. La aparición de los dispositivos móviles no permite suponer que su uso vaya a reemplazar las clases y los encuentros docentealumno. Algunos sostienen que no será así, pero seguramente va a enriquecer sobre todo lo que tiene que ver con la accesibilidad a la información y la formación de grado, la accesibilidad a informes, videos, bibliografía, y la posibilidad de realizar videoconferencias con pares de carreras en otros lugares del país, e incluso en otros lugares del mundo. Se puede dar la clase simultáneamente con otros docentes. Se pueden hacer muchísimas cosas solamente con los teléfonos celulares. Pareciera estar arrancando una nueva generación en lo que es educación superior a partir de estas opciones y estamos en los comienzos. Los informantes se mostraron totalmente de acuerdo, involucrados en el tema y muy optimistas en que va a enriquecer las prácticas docentes universitarias.

\section{DISCUSIÓN}

Para remarcar algunas cuestiones de importancia que intentan promover la discusión y el debate sobre la temática abordada se propone esta síntesis de apreciaciones emergentes del estudio, siendo conscientes de que se han abierto más interrogantes que respuestas a los desafíos propuestos inicialmente. Han sido 
demasiadas y veloces las transformaciones que se han generado por el impacto tecnológico en nuestras vidas y por el fenómeno de la globalización. Más profundos y acelerados han sido los cambios que han generado los dispositivos móviles en poco más de una década, provocando que en la actualidad existan más tarjetas de telefonía celular que habitantes en todo el planeta. Hoy el poder de los teléfonos inteligentes que supera al de la mayoría de aquellas computadoras que regalaba el Estado, hace obsoletas e innecesarias las políticas que impulsaron esos programas. Ya casi el 100 $\%$ de los jóvenes tienen en sus manos un poderoso dispositivo con acceso a Internet y a un gran número de aplicaciones (Anderson y Jiang, 2018). Tenemos la tecnología literalmente al alcance de las manos de todos. El problema es qué hacemos con ello (Bertone, Filippi, Lafuente, Ballesteros, Lafuente, Pérez y Mansilla, 2018).

Creemos que la investigación ha demostrado la percepción de todos los encuestados o entrevistados sobre el enorme potencial que supone esta realidad, sin poder definir aún cómo sacar verdaderamente provecho de ello. Nos hemos acercado a la estimación de la cantidad de docentes y alumnos que ya utilizan sus celulares para mejorar su rendimiento en la universidad. Identificamos el tipo de herramientas y aplicaciones que utilizan en su vida personal, el contraste con su uso en la actividad educativa y la frecuencia con que recurren a los dispositivos móviles. También detectamos el estado y la disponibilidad de redes y equipamiento de los que dispone la universidad dentro de sus instalaciones y las limitaciones y dificultades que enfrenta para acelerar el proyecto tecnológico, junto con las aspiraciones tanto de alumnos como docentes y directivos, en cuanto al tipo de plataforma y disponibilidad de recursos de los que esperan disponer en los próximos años.

Detectamos dentro del estudio algunas cuestiones que nos ayudarán a visualizar en mayor profundidad el estado, la relación y el uso de los dispositivos móviles en la universidad, que referimos en los siguientes párrafos.

Es bajo el uso de dispositivos móviles en la universidad a pesar de que casi la totalidad de alumnos y docentes participantes del estudio dicen tener teléfonos inteligentes con acceso a Internet. Se identifican diferentes habilidades entre docentes y alumnos para la apropiación y el manejo de los dispositivos móviles ("brechas generacionales"). Se puede deducir que no se han desarrollado suficientes aplicaciones, ya que el trabajo muestra que se aprovechan los celulares principalmente para acceder a las redes sociales y para la búsqueda de información y lectura de noticias en diarios digitales. La encuesta revela el WhatsApp con el mayor nivel de utilización, junto con la navegación en Internet, para los dos sectores.

Es bajo todavía el porcentaje de docentes que emplean aplicaciones y herramientas que podrían contribuir a la utilización de dispositivos dentro de la universidad, inclusive el campus virtual. Pocos alumnos consultados en los grupos o en las entrevistas refieren haber tenido experiencias de educación a distancia y sólo algunos profesores indican haber tomado y/o dictado cursos en esa modalidad.

Los entrevistados acuerdan que, en el corto plazo, habrá una gran difusión de la educación virtual o mediada por tecnologías de la información y la comunicación. 
En la encuesta sólo el $2 \%$ de los docentes y alumnos afirmaron que "no hace falta el uso de dispositivos móviles para mejorar las clases en la universidad". Por lo cual se puede deducir que el $98 \%$ cree que es particularmente importante (o al menos necesario), el aprovechamiento de los celulares para mejorar la calidad de las clases en la universidad.

\section{Usar dispositivos móviles como estrategia educativa digital}

En la opinión de los alumnos consultados sobre la calificación del uso de sus teléfonos para las actividades universitarias, el $93 \%$ lo consideran entre bueno a excelente. Lo mismo sucede con los docentes para su actividad académica, llegando al $77 \%$ como se muestra en el siguiente gráfico:

Figura 8. Calificación en el uso de dispositivos móviles

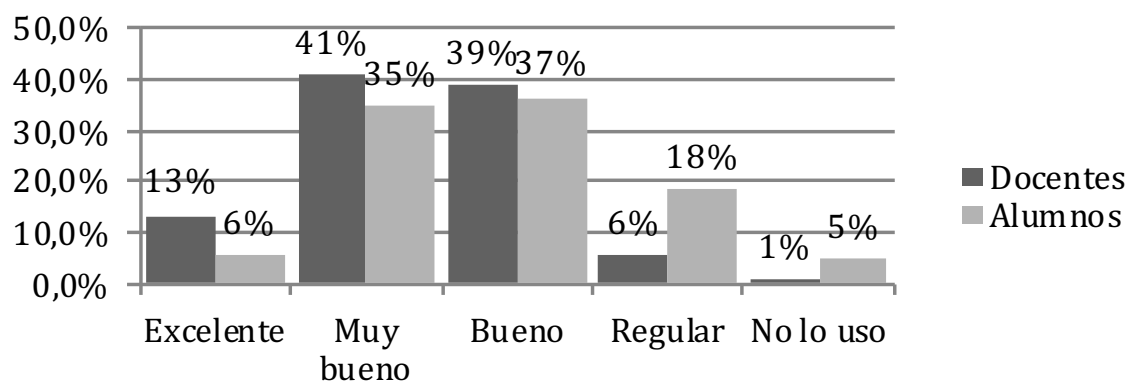

Sin embargo, al consultar cuáles eran las aplicaciones que más utilizaban los alumnos en sus dispositivos móviles, ni en las encuestas, donde destacaron el uso de redes sociales y las búsquedas en Internet, ni en los FG, donde se preguntó específicamente, hubo referencias a actividades directamente vinculadas al proceso de aprendizaje. Esta es otra evidencia empírica sobre la falta de aprovechamiento de estos recursos y la pertinencia de estudios que exploren las razones en sintonía con el objeto de estudio de esta investigación. 
Figura 9. Usos cotidianos de smartphones

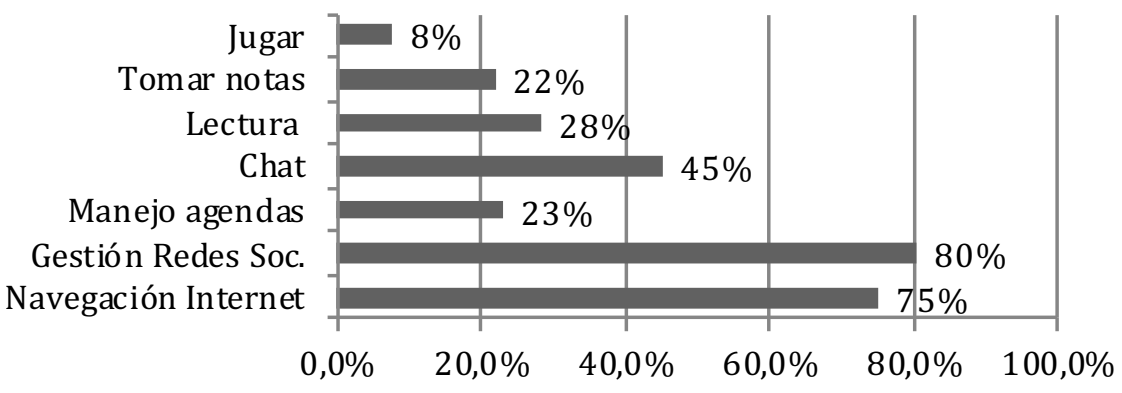

Figura 10. Usos de smartphones para la actividad educativa

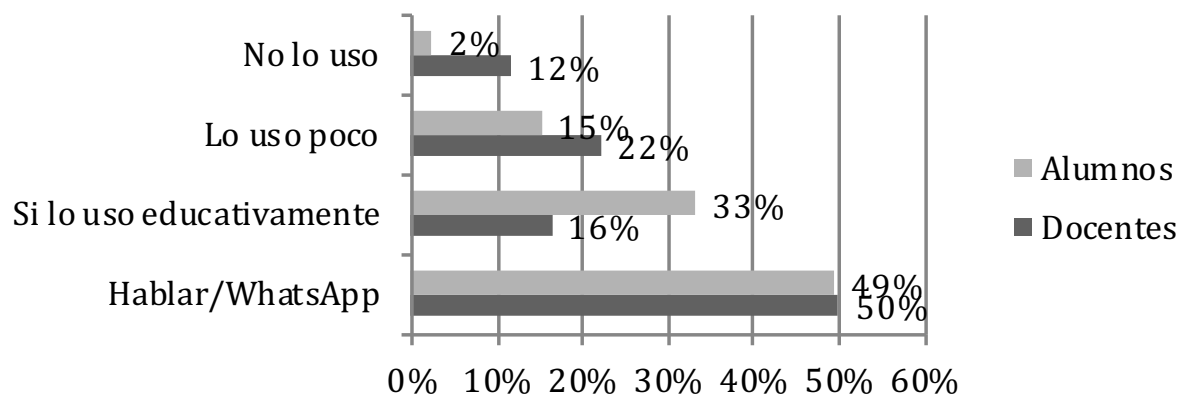

La tenencia de equipos con gran potencial de procesamiento permite focalizar el aprendizaje sobre el alumno, con dinámicas que se facilitan, simplifican o sólo son posibles con los dispositivos móviles.

Si bien es muy importante la adecuación al tipo de carrera, podemos indicar, entre tantos otros, diez posibles usos de celulares para impulsar una estrategia educativa:

1. Comunicación: a través de correos electrónicos, WhatsApp, telefonía.

2. Complemento: uso de aplicaciones de cálculos, tablas, laboratorios virtuales, noticias.

3. Registro: uso de Word, grabadora, fotografía, filmadora.

4. Exploración: uso de buscadores, Google Forms, encuestador en línea.

5. Colaboración: sincrónica o asincrónica con el resto de los alumnos o con otras personas. 
6. Escucha y visualización: de audio y video.

7. Acceso Virtual: a campus virtuales.

8. Interactividad: por medio de aplicaciones como el Mentimeter.

9. Juegos educativos: a través de estrategias de gamificación.

10. Producción de contenidos y aplicaciones específicas por materia.

Se describen también algunas sugerencias de acciones de contexto que permitirán un mayor aprovechamiento de los dispositivos móviles como estrategia didáctica para actualizar la propuesta educativa a las exigencias del cambio tecnológico y la preocupación por la mejora continua:

1. Afianzar la política estratégica en el área tecnológica.

2. Reconvertir el 30 \% permitido en la Resolución del Ministerio de Educación Nro. 2641/17 de todas las materias de todas las carreras al formato virtual.

3. Promover el mayor uso y capacitar en la gestión del campus virtual a todos los docentes de la universidad.

4. Fortalecer el área técnica y de soporte y ayuda a los docentes para implementar la educación virtual y el uso de dispositivos móviles en sus clases.

5. Elaborar planes estratégicos que aceleren la implementación de celulares en las aulas.

6. Profundizar la estrategia e incrementar las inversiones en el tendido de fibras ópticas y el equipamiento necesario que mejore sustancialmente la conectividad y el acceso a internet en todos los espacios del campus de la universidad.

7. Crear mayor cantidad de espacios y momentos de encuentro y capacitación donde los docentes reciban los conocimientos pertinentes.

8. Capacitación previa y certificación de competencias digitales en todos los docentes de la universidad.

9. Generar experiencias piloto (leading case) en el desarrollo de educación con el uso de tecnologías a través de dispositivos móviles, primero con materias y después con carreras, que luego puedan extrapolarse a toda la estructura.

10. Implementar un modelo de gestión de la calidad que permita garantizar la definición de una estrategia clara y realizar el seguimiento de la propuesta.

11. Dar continuidad a la investigación para observar la evolución que producirán las acciones de mejora en el área tecnológica.

12. Diseñar dentro de la universidad un área destinada a elaborar estrategias educativas focalizadas en el uso de teléfonos inteligentes, educación virtual y gestión de tecnologías en comunicación.

En sintonía con los objetivos propuestos, el trabajo ha permitido observar uso, frecuencia, volumen y tipo de aplicación que da la población investigada a sus dispositivos móviles; e indagar sobre las políticas y la infraestructura de la 
plataforma tecnológica, el contexto que los enmarca y las posibles acciones para un mayor aprovechamiento de los mismos (Mangisch, 2018).

Surgen a la vez algunas inquietudes que no hemos abordado en este trabajo que pueden ser motivo de futuros estudios:

- ¿Cuál es la razón por la que algunos docentes prohíben el uso de dispositivos móviles en sus cátedras?

- ¿Cuál es el perfil de docente necesario para la implementación de actividades virtuales?

- ¿Cuál es la preparación específica que requiere un docente para utilizar dispositivos móviles en el aula?

- ¿Cuáles son las políticas que debieran implementar las universidades para promover el desarrollo de experiencias educativas sobre bases tecnológicas?

- ¿Es posible sostener una propuesta "tradicional" de educación en la universidad con los cambios en ciernes?

- ¿Hay que incorporar otro tipo de actores para impulsar propuestas innovadoras con el uso de las TIC en la universidad?

- ¿Están las autoridades dispuestas a promover los cambios que parece demandar el futuro a la universidad?

- ¿Tienen los docentes posibilidades de liderar los cambios que promueve el impacto tecnológico?

- ¿Cuáles deberían ser las estrategias y los contenidos para la capacitación y formación de los docentes en estas nuevas tecnologías?

- ¿Qué debiera hacer la universidad si los cambios epocales obligan a cambiar el paradigma?

\section{CONCLUSIÓN}

El proceso de aprendizaje ha cambiado en los últimos años y se ha vuelto dinámico y continuo, impulsado particularmente por la acelerada evolución de la tecnología, produciendo una transformación inevitable en los métodos de aprendizaje, especialmente en la universidad (Courts y Tucker, 2016). Es un cambio que requiere una transformación digital completa (Sein Echaluce, Fidalgo Blanco y García Peñalvo, 2019). Hace falta adecuar la formación de los actuales universitarios en sintonía con las exigencias de los futuros trabajos y en concordancia con los contextos tecnológicos en los que ya hoy se mueve toda la sociedad. Resulta imperioso diseñar e implementar nuevos programas innovadores de educación y capacitación que se adapten mejor a estas exigencias.

Los dispositivos móviles (como hemos visto en nuestro trabajo) permiten acceder a una inmensa, variada y actualizada cantidad de información y conocimiento en forma inmediata (Deloitte, 2017). Casi toda la población de docentes y alumnos posee un teléfono inteligente con acceso a Internet y parece impensable prescindir 
de él en la vida privada (Russo, Bergami y Morandin, 2017). Por lo cual, ya no hay que preocuparse por la disponibilidad de la herramienta como una limitación para su aprovechamiento.

Por los datos aportados en la investigación concluimos que las universidades ya no necesitarían afectar tanto esfuerzo a la compra de computadoras o el armado de laboratorios de informática para permitir a sus estudiantes y docentes acceder masivamente a la valiosa e imprescindible información que se encuentra en Internet, o para interactuar con el mundo académico con el aprendizaje y gestionar el conocimiento. Por lo mismo parece imperativo priorizar en sus planes el uso de estas nuevas tecnologías privilegiando al estudiante como centro del proceso de enseñanza y aprendizaje (Conde, García Peñalvo, Alier y Piguillem, 2013)

Desde un punto de vista estrictamente técnico, resolviendo la conectividad (Van Dijck, 2016), ya casi no existen más barreras tecnológicas que condicionen a las universidades para impulsar una transformación profunda en la forma de encarar los procesos de enseñanza y aprendizaje, aprovechando a los dispositivos móviles para el diseño de una renovada estrategia educativa. Sólo hace falta que, si no lo hicieron aún, los docentes y los que dirigen las universidades se animen a hacerlo (Universia, 2018).

Las propuestas de mejora que sugerimos en este trabajo pueden servir como inspiración o disparador para aquellos que acepten el desafío y aspiren a ser innovadores protagonistas del cambio, ayudando a orientar así el desarrollo de las nuevas tecnologías al servicio del hombre.

\section{REFERENCIAS}

Adell Segura, J. y Castañeda Quintero, L. (2010). Los Entornos Personales de Aprendizaje (PLEs): Una nueva manera de entender el aprendizaje. En R. Roig Vila y M. Fiorucci (Ed.), Claves para la investigación en innovación y calidad educativas. La integración de las Tecnologías de la Información y la Comunicación y la Interculturalidad en las aulas. Alcoy: Marfil - Roma TRE Universita degli studi Recuperado de: http://cent.uji.es/pub/sites/cent/files/ Adell Castaneda 2010.pdf

Aguiar, B. O., Velázquez, R. M., y Aguiar, J. L. (2019). Innovación docente y empleo de las TIC en la Educación Superior. Revista ESPACIOS, 4O(02). Recuperado de https://www.revistaespacios.com/ a19v40n02/19400208.html
Almenara, J. C., y Osuna, J. B. (2015). Nuevos retos en tecnología educativa. Madrid: Editorial Síntesis.

Anderson, M., y Jiang, J. (2018). Teens, social media \& technology. Washington, DC. Blog Pew Research Center. Recuperado de www.pewinternet.org/2018/05/31/ teens-social-media-technology-2018/

Arkorful, V., y Abaidoo, N. (2014). The role of e-learning, advantages and disadvantages of its adoption in higher education. International Journal of Education and Research 2(12). Recuperado de https://www.ijern.com/journal/2014/ December-2014/34.pdf

Bender, W., Kaner, Ch., Cornish, J., y Donahue, D. (2012). Learning to Change the World: The Social Impact of One Laptop Per Child. Boston: St. Martin's Press. 
Bertone, R., Filippi, J. L., Lafuente, G. J., Ballesteros, C. A., Lafuente, G., Pérez, D., ... Mansilla, A. (2018). Dispositivos móviles como instrumento facilitador del aprendizaje. 1073-1077. Recuperado de http://sedici.unlp.edu.ar/ handle/10915/68388

Cassany, D. (2013). Sociedad de conocimiento, tecnología y comunicación. Google Books. Recuperado de https:// cutt.ly/Ueu6R3k

Conde, M.Á., García Peñalvo, F. J., Alier, M., y Piguillem, J. (2013). The implementation, deployment and evaluation of a mobile personal learning environment. Journal of Universal Computer Science, 19(7), 854-872.

Courts, B., y Tucker, J. (2016). Using Technology to Create a Dynamic Classroom Experience. Journal of College Teaching \& Learning (TLC), 9(2), 121. doi: https://doi.org/10.19030/tlc.v9i2.6907

Deloitte (2017). Informe sobre Consumo móvil en Argentina. Los móviles prueban ser indispensables en un mundo "siempre" conectado. Buenos Aires: Blog Deloitte. Recuperado de https://cutt.ly/2eu6EHj

Fernández Muñoz C., y García García F. (2016). The use of mobile phones as tools for access and knowledge exchange by students in Spain when studying. Revista Prisma Social. No Especial 1, 190-208. Madrid. Recuperado de http://revistaprismasocial.es/article/ view/1318/1382

Fontdevila, P. (2011). Inclusión digital y calidad educativa - El Programa Conectar Igualdad entre 2010 y 2011. Buenos Aires: Editorial Eduntref.

Henríquez Ritchie, P., Organista Sandoval, J., y Lavigne, G. (2013). Nuevos Procesos de interactividad e Interacción social: Uso de Smartphones por Estudiantes y Docentes Universitarios. Revista Electrónica Actualidades Investigativas en Educación. Costa Rica, 3(13).
Recuperado de http://www.redalyc.org/ articulo.oa?id $=44729878012$

Hernández Ramos, J. P., y Martínez Abad, F. (2017). El empleo del Smartphone en el aula universitaria como herramienta para la gamificación de la docencia. Blog Conference Edunovatic 2017, 2nd Virtual International Conference on Education, Innovation and ICT. Recuperado de https://cutt.ly/Deu6ylV

Kostakos, V. (2018). Smartphones for science. Blog The university of Melbourne. Australia. Recuperado de https://cis. unimelb.edu.au/research/groups/ interaction-design/projects/smartphones

Lagunes Domínguez, A. (2019). Prospectiva hacia el Aprendizaje Móvil en Estudiantes Universitarios. Formación Universitaria, 1O(1), Recuperado de https://scielo. conicyt.cl/scielo.php? script $=$ sci arttext\&pid=So718-50062017000100011

López Hernández, F. A., y Silva Pérez, M. M. (2016). Factores que inciden en la aceptación de los dispositivos móviles para el aprendizaje en educación superior. Estudios Sobre Educacion, 30, 175-195. doi: https://doi. org/10.15581/004.30.175-195

Lozano Ramírez, M. C. (2019). Aprendizaje social basado en el uso de dispositivos móviles. Barcelona. RITI, Revista de Investigación en Tecnologías de la Información, 7(13), 48-52. Recuperado de http://www.riti.es/ojs2018/inicio/index. $\mathrm{php} / \mathrm{riti} /$ article/view/121

Ma'azer Al Fawareh, H., y Jusoh, D. (2017). The Use and Effects of Smartphones in Higher Education. Blog Northern Border University, Rafha. Recuperado de https:// cutt.ly/Deu539T

Mangisch, G. (2018). El uso de los dispositivos móviles de los principales actores del proceso de enseñanza y aprendizaje en la universidad. Instituto LidInCom UCCuyyo. Recuperado de http://ffh.uccuyosj.edu.ar/images/ Informe final DM 11 11.pdf 
Mao, J., Ifenthaler, D., Fujimoto, T., Garavaglia, A., y Rossi, P. G. (2019). National Policies and Educational Technology: a Synopsis of Trends and Perspectives from Five Countries. Blog Tech Trends 63, 284. doi: https://doi. org/10.1007/s11528-019-00396-0

Márquez, I. (2019). Móviles 24/7. El teléfono móvil en la era de la hiperconectividad. Barcelona: Editorial UOC. Recuperado de https://www.researchgate.net/ publication/331487571 Resena Moviles 247 El telefono movil en la era de la hiperconectividad de Israel Marquez Barcelona Editorial UOC 2018

Pinos Paredes, N. (2018). Uso del celular como distractor del proceso enseñanza aprendizaje. Investigación, Vinculación, Docencia y Gestión, 3(4), 166-171 Recuperadodehttps://www.researchgate. net/publication/329914655 Uso del telefono celular como distractor del proceso ensenanza - aprendizaje

Rivoir, A. (2013). Plan Ceibal e Inclusión Social Perspectivas interdisciplinarias. Plan Ceibal/Udelar. Montevideo, Uruguay.
Russo, M., Bergami, M., y Morandin, G. (2017). Surviving a Day Without Smartphones. MIT Sloan Management Review, 59(2), 8. Recuperado de https:// sloanreview.mit.edu/article/surviving-aday-without-smartphones

Schlender, B., y Tetzeli, R. (2016). El libro de Steve Jobs. Barcelona: Malpaso.

Sein Echaluce, M. L., Fidalgo Blanco, Á., y García-Peñalvo, F. (2019). Innovative Trends in Flipped Teaching and Adaptive Learning. IGI Global. doi: 10.4018/978-15225-8142.

Solano Barcenas, O. S. (2015). Revolución tecnológica y democracia del conocimiento. Por una universidad innovadora. Misión Jurídica. Revista de derecho y ciencias sociales, 8(9), 319-322. Recuperado de https://dialnet.unirioja. es $/$ servlet/articulo?codigo $=5645580$

Universia (2018). IV Encuentro de Rectores. Universidad, Sociedad y Futuro. Declaración de Salamanca. Universidad de Salamanca. Salamanca. Recuperado de https://universiasalamanca2018.com

Van Dijck, J. (2016). La cultura de la conectividad. Una historia crítica de las redes sociales. México: Siglo XXI Editores.

\section{PERFIL ACADÉMICO Y PROFESIONAL DE LOS AUTORES}

Gustavo Carlos Mangisch Moyano. Graduado en las carreras de sistemas y de administración y tiene un máster y tres doctorados vinculados a las ciencias sociales, la administración, la comunicación y la educación. Es director de un Instituto de investigación, una maestría y varias iniciativas vinculadas al tema de la gestión de las nuevas tecnologías en comunicación en la Universidad Católica de Cuyo. Líneas de investigación: liderazgo, innovación y TIC.

E-mail: gmangisch@uccuyo.edu.ar

María del Rosario Mangisch Spinelli. Graduada en las carreras de enseñanza del inglés, en gestión educativa y máster en educación. Es investigadora en la UCCuyo y docente del nivel primario, secundario y universitario. Líneas de investigación: liderazgo, innovación y TIC.

E-mail: ro.mangisch@gmail.com 
Dirección:

Av. José Ignacio de la Roza 1516,

J5400 San Juan. (Argentina)

Fecha de recepción del artículo:20/06/2019

Fecha de aceptación del artículo: 31/08/2019

Fecha de aprobación para maquetación: 06/10/2019 